\title{
Transposition Flap for Cutaneous Lesion Coverage in Dermatomyositis
}

\author{
Jaehyun Bae (D), Dongkeun Jun (D), Jeenam Kim (D), Hyungon Choi (D), Donghyeok Shin (D), Myungchul Lee (D) \\ Department of Plastic and Reconstructive Surgery, Konkuk University Medical Center, Seoul, Korea
}

\begin{abstract}
Dermatomyositis (DM) is an autoimmune inflammatory myopathy with some cutaneous manifestations. We present the case of a patient with a bone-depth skin ulceration on the elbow that was well healed with a transposition flap using a dorsal interosseous artery perforator. A 56-year-old man reported difficulties when climbing stairs and diffuse pain of unclear origin throughout his body that started 2 months previously. The patient was diagnosed with DM, and the department of plastic and reconstructive surgery was consulted for the management of an ulcerative skin lesion on the left elbow. The dorsal interosseous artery was first detected by vascular Doppler ultrasonography, and a transposition flap was designed including the pedicle. The flap was transposed to cover the bone exposure over the olecranon. At 4 weeks postoperatively, complete healing of the wound was observed without complications, and the patient had full range of motion of the elbow without pain. Despite major developments in the medical treatment of DM, ulcerative cutaneous lesions remain difficult to treat. Our experience suggests that the use of a transposition flap including the dorsal interosseous artery is suitable for the treatment of ulcerative lesions around the elbow in DM patients.
\end{abstract}

Keywords: Dermatomyositis; Elbow joint; Pedicled flap

\section{Introduction}

Dermatomyositis (DM) is an autoimmune inflammatory myopathy with some cutaneous manifestations, including Gottron papules, heliotrope rash, and violaceous rash. In $30 \%$ of patients with DM, the onset of cutaneous disease can come several months before the appearance of myositis, whereas muscle involvement is followed by cutaneous manifestations in $10 \%$ of patients, and the remaining $60 \%$ of patients have roughly simultaneous onset of cutaneous symptoms and muscle involvement [1]. In most patients, DM starts with symmetrical proximal muscle weakness. As a result, patients may have difficulty climbing stairs, or they may experience dysphagia if DM affects skeletal muscles in the oropharynx and upper esophagus.

$\mathrm{DM}$ is diagnosed on the basis of one or more of the skin symptoms (Gottron papules, heliotrope rash, and violaceous rash), with four or more of the following findings: muscle weakness, muscle pain, elevated serum levels of muscle-derived enzymes, electromyogram (EMG) findings of myositis, anti-Jo-1 antibodies, systemic inflammatory findings, and pathological findings on muscle biopsy.

There is no universally accepted standard treatment of DM, but corticosteroids are the main treatment of choice, and immunosuppressants are also used in some cases. The goal of treatment is to increase muscular strength, thereby improving the patient's daily life, and to eliminate extramuscular manifestations of the disease. Though surgical treatment is beneficial for localized lesions, it is not commonly performed on DM patients due to the high rates of recurrence, infection, sinus formation, and difficult

\section{Case Report}

Received: October 2, 2020

Revised: October 13, 2020

Accepted: October 13, 2020

\section{Corresponding author:} Myungchul Lee, M.D.

Department of Plastic and Reconstructive Surgery, Konkuk University Medical Center, 120-1 Neungdong-ro, Gwangjin-gu, Seoul 05030, Korea

Tel: +82-2-2030-8191

Fax: +82-2-2030-5249

E-mail: 20150136@kuh.ac.kr

This is an Open Access article distributed under the terms of the Creative Commons Attribution Non-Commercial License (https://creativecommons.org/licenses/by-nc/4.0/) which permits unrestricted non-commercial use, distribution, and reproduction in any medium, provided the original work is properly cited.

C 2020 Korean Wound Management Society 
wound healing [2]. Systemic treatment of DM, which may include corticosteroids, immunosuppressive agents, and/or radiation can aggravate these risks. Thus, poor wound healing can be caused by abnormal conditions of the surrounding tissues, and the systemic effects of the corticosteroids and immunosuppressive agents.

Here, we report a case involving a DM patient who presented with a bone-depth skin ulceration of the elbow. In this case, we covered the wound with a transposition flap using a dorsal interosseous artery perforator. The study protocol conformed to the ethical guidelines of the Declaration of Helsinki, and verbal informed consent was obtained from the patient according to the decision of the Institutional Review Board of Konkuk University Medical Center (IRB exemption No. 202009-047).

\section{Case}

A 56-year-old man with no noteworthy history sought medical care for difficulty in climbing stairs and a diffuse pain of unclear origin throughout his body that had started 2 months ago. At a local clinic, he was diagnosed with a simple cold and treated conservatively for pain. However, his shoulder pain became worse, he developed dyspepsia, and his weakened thigh strength did not improve; upon further consideration, these were recognized as proximal muscle weakness symptoms. He visited the department of anesthesiology and received painkillers for systemic myalgia 1 month ago. Around that time, he identified an ulcerative lesion on his left knee, after which his pace of walking slowed. When starting to move from rest, he felt discomfort and pain, which decreased as he moved.

Ulcerative and painful skin symptoms, included dermatitis and rash, occurred on both forearms and hands, including the metacarpophalangeal joint, wrist, and elbow, and were aggravated 2 weeks previously. He was admitted to the department of dermatology for treatment of the dermatitis and rash with prednisolone $0.2 \mathrm{mg} / \mathrm{kg} /$ day. EMG testing was conducted and the Medical Research Council sum score, an indicator of overall muscle strength, was 48 of 60 . The findings supported active myopathy in the proximal muscles, with a clinical impression of inflammatory myopathy.

As the ulcerative and inflammatory skin lesion on his left elbow showed no improvement, the patient was referred to the department of plastic and reconstructive surgery for professional wound management of the ulcerative wound. He had
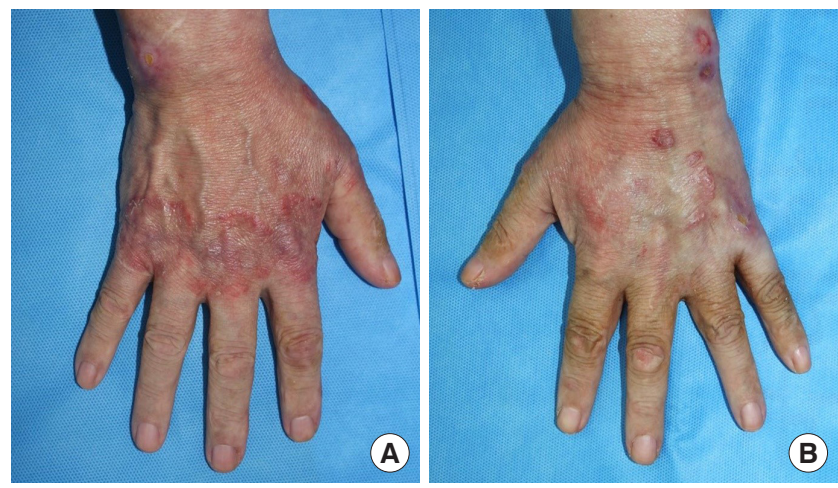

Fig. 1. Gottron papules on bilateral hands of a dermatomyositis patient. Typical erythematous to violaceous papules were presented on the metacarpophalangeal joints of the right hand.

Gottron papules-erythematous to violaceous papules on the dorsal side of the various joints of hands-, a heliotrope rash, and a violaceous rash; therefore, the authors suspected an autoimmune disease and performed a muscle biopsy at the right shoulder in consultation with the department of rheumatology (Fig. 1). The histology results, including numerous degenerating and regenerating myofibers with perifascicular degeneration, several atrophic myofibers, and perivascular lymphocytes, were consistent with DM. The serum levels of enzymes derived from muscle tissue were as follows: aldolase, $16.8 \mathrm{IU} /$ L; alkaline phosphatase, $140 \mathrm{IU} / \mathrm{L}$; creatine phosphokinase (CK), $78 \mathrm{IU} / \mathrm{L}$; and lactate dehydrogenase (LDH), $944 \mathrm{IU} / \mathrm{L}$. In addition, antinuclear antibodies (ANA) were positive, but anti-Jo- 1 antibodies were negative. Based on the above information, he was diagnosed with DM.

The rheumatologists recommended that additional exams were needed after diagnosis of DM, because DM over middle age is often accompanied by paraneoplastic syndrome. Thus chest computed tomography, esophagogastroduodenoscopy, and colonoscopy were performed to screen for paraneoplastic syndrome, and there were no abnormal findings. Upon physical examination, the bone-depth ulcer measured $2 \times 1 \mathrm{~cm}$ and was positioned at the tip of the olecranon. The surrounding tissue was reddish and had pus-like discharge over the swelling (Fig. 2). Magnetic resonance imaging (MRI) was conducted to accurately assess the wound of the left elbow. The MRI findings showed rupture of the olecrenon bursa, infectious olecrenon bursitis, and infection involvement in the adjacent muscles, including the triceps and anconeus muscles (Fig. 3).

The inflammatory ulcer was treated by drainage with debridement, and the antibiotic Ceftriaxone was prescribed based on the results of the wound culture, Morganella morganii. 


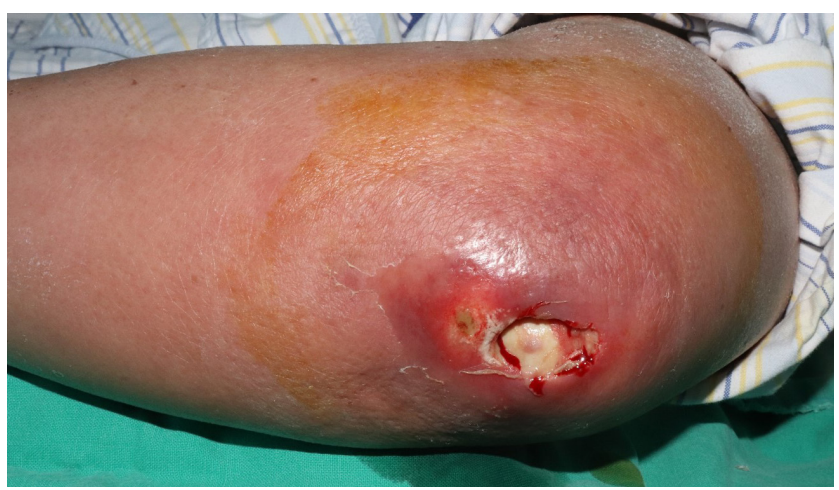

Fig. 2. The initial wound. An ulcerative skin lesion on the left elbow. The olecranon process of ulna was exposed through the wound.
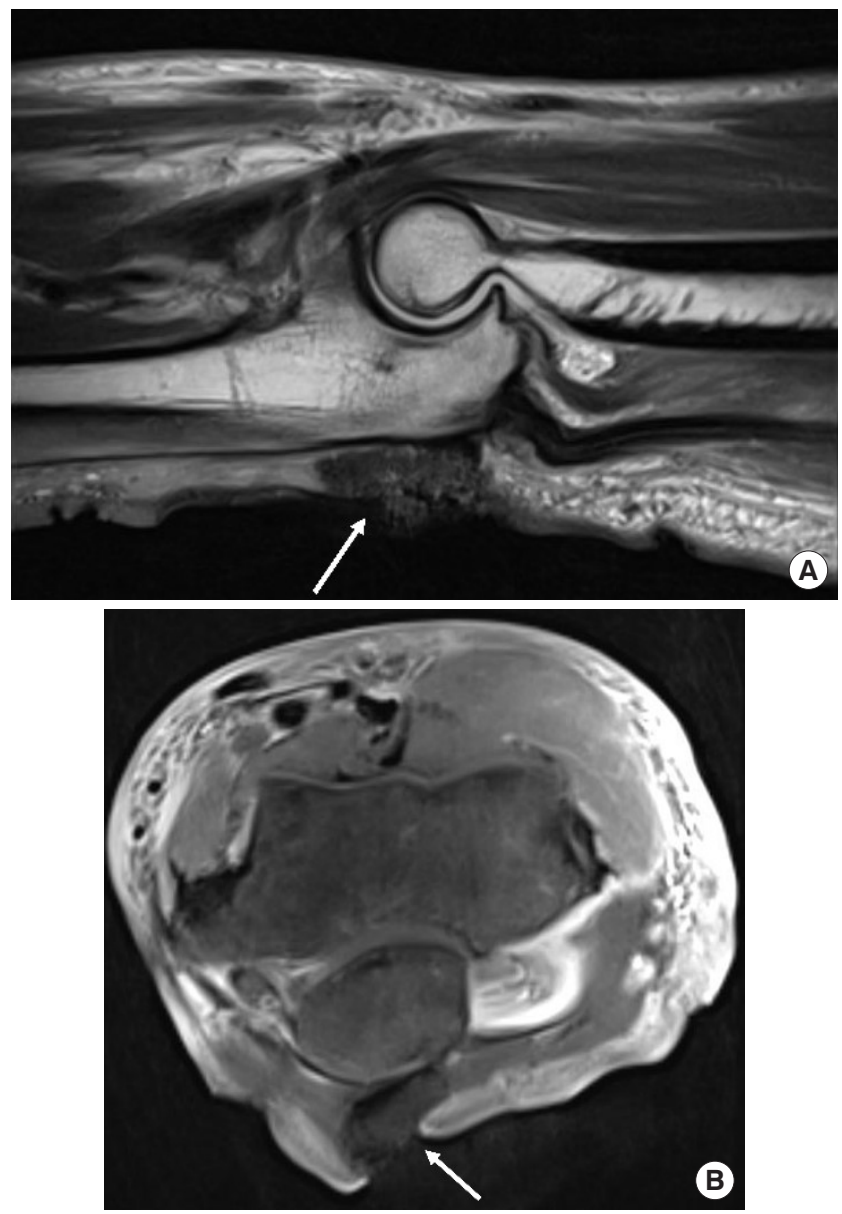

Fig. 3. Imaging study of the ulcerative wound. (A, B) Magnetic resonance images of the lesion. The sagittal and axial view images showed infectious olecranon bursitis and rupture of the olecranon bursa (arrow).

After the inflammation subsided, negative-pressure wound therapy was applied three times, at 3-day intervals. When the

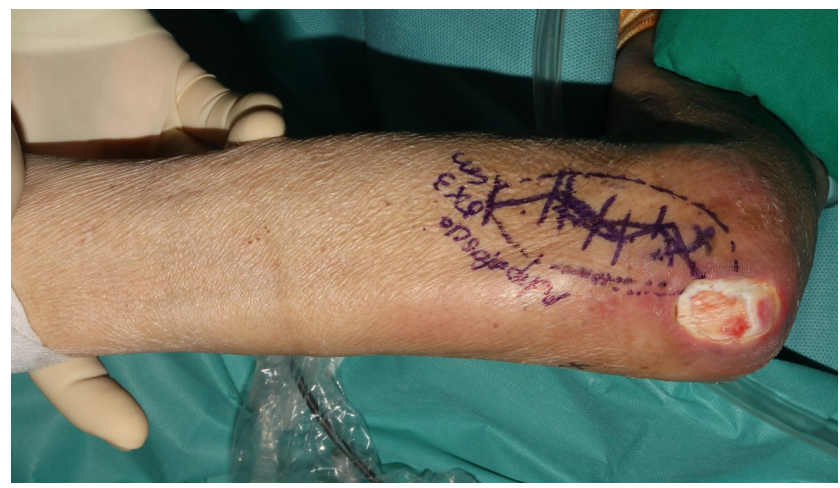

Fig. 4. The initial operative plan to cover the defect. The initial plan for an adipofascial flap was changed to a transposition flap, since severe atrophy was noted in the subcutaneous layer and very scant tissue was available.

wound bed was prepared for coverage, an adipofascial flap was planned (Fig. 4). However, the surrounding subcutaneous tissue and the fascia showed atrophic changes, so the surgical plan was modified to a pedicled transposition flap. The dorsal interosseous artery was detected by vascular Doppler ultrasonography at first, and the transposition flap was designed to include the pedicle (Fig. 5). The flap was transposed to cover the bone exposure over the olecranon.

Postoperatively, a long arm splint was applied for 2 weeks, and steroid and immunosuppressant treatment was delayed in consultation with the department of rheumatology. At 4 weeks postoperatively, complete healing of the wound without complications was observed, and the patient had full range of motion of the elbow without pain (Figs. 6, 7). Starting 4 weeks after surgery, the patient took prednisolone and an additional immunosuppressant (tacrolimus). The rheumatology team slowly tapered the dosage of medication based on follow-up laboratory results and clinical manifestations.

\section{Discussion}

DM primarily involves a humoral immune mechanism that causes microvascular disease and ischemia in muscle cells. Autoantibodies are used as laboratory evidence of myositis. The ANA positivity rate is about $60 \%$. Anti-Jo-1 antibodies have high specificity, but are only positive in approximately $20 \%$ of cases. Anti-Mi- 2 antibody positivity can also be helpful for making the diagnosis. Myositis causes muscle destruction; therefore, serum CK, LDH, aspartate aminotransferase, alanine aminotransferase, and myoglobin levels can increase.

EMG is conducted to distinguish myopathic causes of weak- 


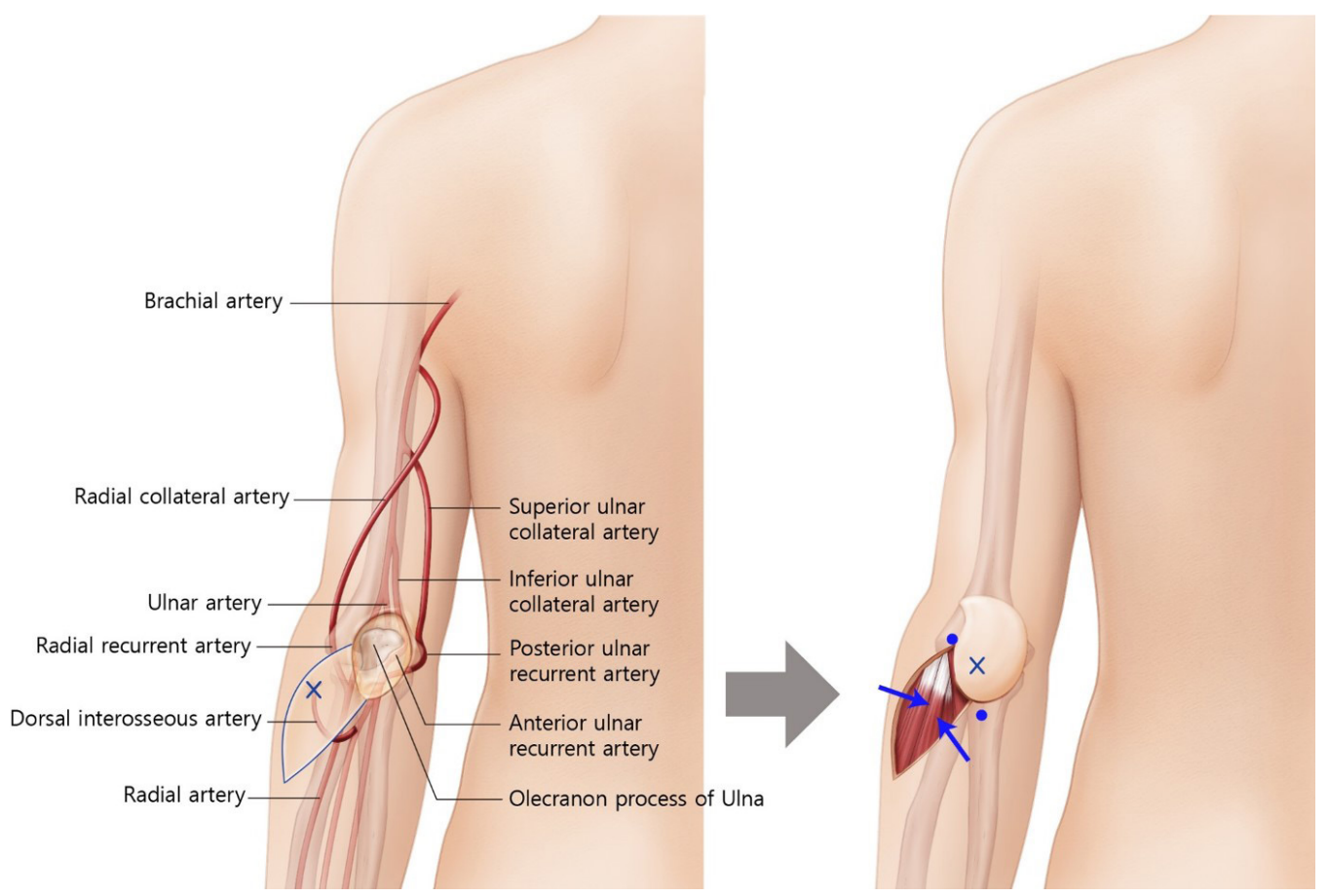

Fig. 5. Illustration of operative procedures. A transposition flap to cover the defect over the olecranon was designed including the dorsal interosseous artery (X, location of the dorsal interosseous artery perforator pedicle; blue dots, opposing tissues after transposing the flap; blue arrows, skin and soft tissue advancement for donor site closure).

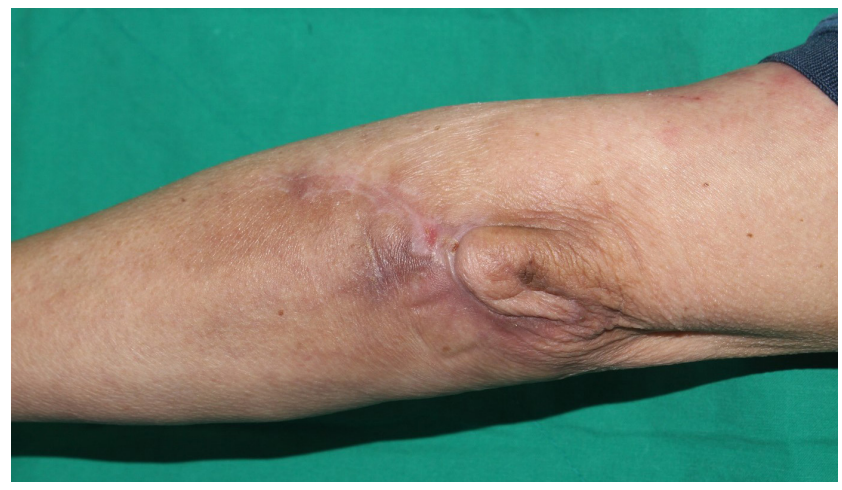

Fig. 6. The healed wound at 4 weeks postoperatively.

ness from neuropathic disorders. Myopathic potential readings are characterized by increased spotaneous activity, such as short durations, low amplitude, polyphasic motor unit action potentials, fibrillation, complex repetitive discharges, and positive sharp waves. To check the presence of muscle destruction, a biopsy is generally performed to evaluate infiltration of inflammatory cells (including lymphocytes) into the deltoids, biceps brachii, or biceps femoris. An important pathologic finding is perifascicular atrophy, which manifests as two to 10 layers of atrophied muscle fibers observed around the muscle fascicle. This is a characteristic finding that enables DM to be diagnosed even without inflammatory cell infiltration. MRI of skeletal muscles can be used to identify areas of severe muscular dystrophy and muscle inflammation with active myositis, fibrosis, and calcification [3]. In adults, the comorbidity rate of malignant tumors such as melanoma, colorectal cancer, and non-Hodgkin lymphoma is about $30 \%$.

Narang et al. [4] reported that cutaneous ulcerations were present in $28 \%$ of 152 patients with DM and that the ulceration had potential associations with autoantibody status. The anti-melanoma differentiation-associated gene 5 antibody is known to be associated with skin ulceration in DM [5]. Cutaneous ulcerations commonly occur over Gottron papules or in areas with the Gottron sign, and often affect the skin over the extensor joint surfaces, although they could be found anywhere [6]. An evaluation of the lesions using objective measurements, such as the Cutaneous Dermatomyositis Disease Area and Severity Index, is useful for checking the severity of the lesions and assessing their response to treatment [7].

In patients with $\mathrm{DM}$, quality-of-life impairment related to skin disease is significant [8]. A preferred method to manage cutaneous manifestations of DM has not yet been established [9-11]. To achieve a satisfactory response of skin manifestations in patients with cutaneous DM, a variety of treatment 

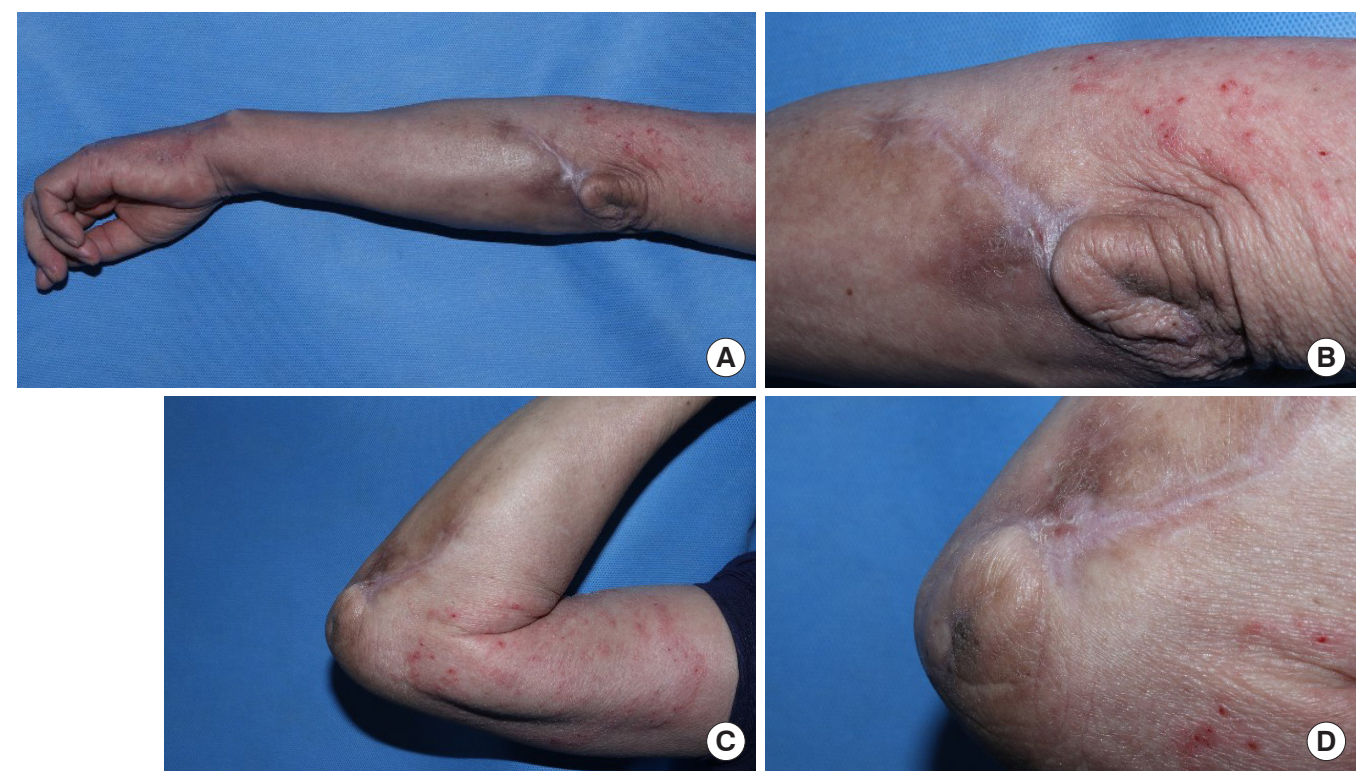

Fig. 7. Range of motion at long-term follow-up. Complete healing of the wound was achieved at 7 months postoperative follow-up (A, B) and a full range of motion was available without limitation (C, D).

approaches may be necessary, including aggressive photoprotection to reduce the exacerbating effects of ultraviolet light, antipruritic agents to manage the associated pruritus, topical corticosteroids for local treatment, and systemic medications aimed at attaining sustained control of the disease [12].

Some patients with mild skin manifestations of DM can achieve sufficient improvement without systemic therapy, but most patients require systemic therapy to suppress cutaneous lesions. In this case, we planned to postpone systemic therapy until complete wound healing of the surgical wound on the patient's left elbow, because we thought that the medications might delay the healing of the wound of the transposition flap coverage. In fact, avoidance of steroid and immunosuppressant drugs is recommended until complete wound healing occurs, because these medications interfere with inflammatory mediators by stabilizing lysosomes within neutrophils, inducing anti-inflammatory proteins, and inhibiting cytokine release and chemotaxis [13]. Furthermore, fibroblast dysfunction, reduced collagen production, angiogenesis, re-epithelialization, and decreased wound tensile strength can be caused by corticosteroids. The mechanisms of corticosteroid-induced downregulation of wound healing can be explained by stimulation of the $\beta$-catenin and c-myc pathways [14]. The deposition of calcium within the skin occurs commonly in juvenile $\mathrm{DM}$, not typically in adult DM, and the most common complication from excision of the calcinosis cutis is poor wound healing caused by abnormal conditions of the surrounding tis- sues, and systemic effects of the corticosteroids and immunosuppressive agents [4].

Most studies dealing with DM mention surgery for subcutaneous calcifications, while few studies have dealt with the surgical treatment of ulcerations. Surgical treatment may be required for painful, infected lesions or ulcerations that interfere with joint function. Herein, we presented a case of coverage using a local transposition flap. The flap contained the dorsal interosseous artery pedicle, was elevated deep to the fascia, and transposed to cover the defect. The perforator of a cutaneous flap based on a vessel supplying the skin has its own vascular territory, called a perforasome. Various new perforator flaps have been reported in the past decade. Local perforator flaps can provide tissues with good color match and contour to ensure optimal tissue replacement and minimal donor-site morbidity, while maintaining function [15]. The choice of a proper perforator close to the defect for pedicled perforator flaps requires expertise and a high level of operative skill.

Although major advances have been made in the medical treatment of DM, ulcerative cutaneous lesions remain difficult to treat. Literature includes few reports of the surgical technique used to cover these ulcerations, which often occur on the elbow. Further studies are needed to find the best therapeutic approach for cutaneous manifestations of DM. Our experience suggests that the use of a transposition flap including the dorsal interosseous artery is suitable for the treatment of ulcerative lesions around the elbow in patients with DM. 


\section{Conflict of interest}

No potential conflict of interest relevant to this article was reported.

\section{ORCID iDs}

Jaehyun Bae https://orcid.org/0000-0001-8716-3109

Dongkeun Jun

Jeenam Kim https://orcid.org/0000-0001-9017-9929

Hyungon Choi

Donghyeok Shin

Myungchul Lee

https://orcid.org/0000-0002-4080-6135

https://orcid.org/0000-0002-3816-1286

https://orcid.org/0000-0002-8450-4411

https://orcid.org/0000-0002-9721-0092

\section{References}

1. Sontheimer RD. The management of dermatomyositis: current treatment options. Expert Opin Pharmacother 2004; 5:1083-99.

2. Welborn MC, Gottschalk H, Bindra R. Juvenile dermatomyositis: a case of calcinosis cutis of the elbow and review of the literature. J Pediatr Orthop 2015;35:e43-6.

3. May DA, Disler DG, Jones EA, et al. Abnormal signal intensity in skeletal muscle at MR imaging: patterns, pearls, and pitfalls. Radiographics 2000;20 Spec No:S295-315.

4. Narang NS, Casciola-Rosen L, Li S, et al. Cutaneous ulceration in dermatomyositis: association with anti-melanoma differentiation-associated gene 5 antibodies and interstitial lung disease. Arthritis Care Res (Hoboken) 2015;67:66772 .

5. Li L, Wang Q, Yang F, et al. Anti-MDA5 antibody as a potential diagnostic and prognostic biomarker in patients with dermatomyositis. Oncotarget 2017;8:26552-64.

6. Cao H, Xia Q, Pan M, et al. Gottron papules and Gottron sign with ulceration: a distinctive cutaneous feature in a subset of patients with classic dermatomyositis and clinically amyopathic dermatomyositis. J Rheumatol 2016;43: 1735-42.

7. Tiao J, Feng R, Bird S, et al. The reliability of the Cutaneous Dermatomyositis Disease Area and Severity Index (CDASI) among dermatologists, rheumatologists and neurologists. Br J Dermatol 2017;176:423-30.

8. Hundley JL, Carroll CL, Lang W, et al. Cutaneous symptoms of dermatomyositis significantly impact patients' quality of life. J Am Acad Dermatol 2006;54:217-20.

9. Oddis CV, Conte CG, Steen VD, et al. Incidence of polymyositis-dermatomyositis: a 20-year study of hospital diagnosed cases in Allegheny County, PA 1963-1982. J Rheumatol 1990;17:1329-34.

10. Iorizzo LJ 3rd, Jorizzo JL. The treatment and prognosis of dermatomyositis: an updated review. J Am Acad Dermatol 2008;59:99-112.

11. Bendewald MJ, Wetter DA, Li X, et al. Incidence of dermatomyositis and clinically amyopathic dermatomyositis: a population-based study in Olmsted County, Minnesota. Arch Dermatol 2010;146:26-30.

12. Quain RD, Werth VP. Management of cutaneous dermatomyositis: current therapeutic options. Am J Clin Dermatol 2006;7:341-51.

13. Levine JM. The effect of oral medication on wound healing. Adv Skin Wound Care 2017;30:137-42.

14. Jozic I, Vukelic S, Stojadinovic O, et al. Stress signals, mediated by membranous glucocorticoid receptor, activate PLC/ PKC/GSK-3 $\beta / \beta$-catenin pathway to inhibit wound closure. J Invest Dermatol 2017;137:1144-54.

15. Saint-Cyr M, Wong C, Schaverien M, et al. The perforasome theory: vascular anatomy and clinical implications. Plast Reconstr Surg 2009;124:1529-44. 